\title{
Significant Genetic Heterogeneity of the SIVmac251 Viral Swarm Derived from Different Sources
}

\author{
Samantha L. Strickland, ${ }^{1,2,{ }^{*}}$ Rebecca R. Gray, ${ }^{1,2, *}$ Susanna L. Lamers, ${ }^{3}$ Tricia H. Burdo, ${ }^{4}$ \\ Ellen Huenink, David J. Nolan, ${ }^{1,2}$ Brian Nowlin, ${ }^{4}$ Xavier Alvarez, ${ }^{5}$ Cecily C. Midkiff, \\ Maureen M. Goodenow, Kenneth Williams, ${ }^{4}$ and Marco Salemi ${ }^{1,2}$
}

\begin{abstract}
Infecting rhesus macaques (Macaca mulatta) with the simian immunodeficiency virus (SIV) is an established animal model of human immunodeficiency virus (HIV) pathogenesis. Many studies have used various derivatives of the SIVmac251 viral swarm to investigate several aspects of the disease, including transmission, progression, response to vaccination, and SIV/HIV-associated neurological disorders. However, the lack of standardization of the infecting inoculum complicates comparative analyses. We investigated the genetic diversity and phylogenetic relationships of the 1991 animal-titered SIVmac251 swarm, the peripheral blood mononuclear cell (PBMC) passaged SIVmac251, and additional SIVmac251 sequences derived over the past 20 years. Significant sequence divergence and diversity were evident among the different viral sources. This finding highlights the importance of characterizing the exact source and genetic makeup of the infecting inoculum to achieve controlled experimental conditions and enable meaningful comparisons across studies.
\end{abstract}

E XPERIMENTAL INFECTION OF rhesus macaques (Macaca mulatta) with simian immunodeficiency virus (SIV) provides a powerful platform for studying the pathogenesis of AIDSrelated neurological disorders and for the development of effective vaccines. ${ }^{1-3}$ The natural course of infection in the SIV / macaque model is similar to that of non-antiretroviral-treated HIV-1 patients. ${ }^{1}$ For example, SIV-infected monkeys often have chronic diarrhea and large decreases in body weight at the time of death. The absolute number of $\mathrm{CD}^{+}$lymphocytes, as well as CD4:CD8 ratio, decreases in the peripheral circulation during the infection. ${ }^{4}$ Additionally, many of the macaques develop a viral-induced meningoencephalitis. ${ }^{1}$

SIVmac251 and SIVsmE660 are the most common pathogenic viral swarms used as inoculum in experimental animal models. This study concentrates on the SIVmac251 viral swarm, which was initially derived from rhesus macaque Mm251-79 infected by inoculation of minced spontaneous lymphoma tissue, and subsequently amplified in monkeyderived cells. ${ }^{5}$ This stock is considered the "original 1991 viral swarm (SIVmac251_1991)." Since that time, different SIVmac251 stocks were obtained. Many resulted by passaging in vitro the original SIVmac251_1991 or by isolating a new swarm from animals infected with the 1991 quasispecies. Other SIVmac251 swarms were passaged in parallel to the SIVmac251_1991 quasispecies (R. Desrosiers, personal communication). However, with a few exceptions, ${ }^{6-8}$ the origin and culture condition of the stock being used as the inoculum in each specific experimental setting are frequently unclear from publications.

The lack of in-depth characterization of these differently derived SIVmac251 swarms, and their potential variability, may introduce a confounding factor in the interpretation and comparison of results across studies. For example, the use of more virulent swarms may underestimate the potential protective efficacy of some immunization strategies., 90 Additionally, inocula with diverse genetic heterogeneity may lead to different outcomes during acute infection due to the number of transmitted variants and/or the presence of viral strains with specific phenotypes (e.g., cellular tropism). Therefore, the objective of the present study was to compare the diversity and analyze the phylogenetic relationships of the different SIVmac251 viral swarms.

\footnotetext{
${ }^{1}$ Department of Pathology, Immunology, and Laboratory Medicine, University of Florida, Gainesville, Florida.

${ }^{2}$ Emerging Pathogens Institute, University of Florida, Gainesville, Florida.

${ }^{3}$ BioInfoExperts, Thibodeaux, Louisiana.

${ }^{4}$ Department of Biology, Boston College, Boston, Massachusetts.

${ }^{5}$ Tulane National Primate Research Center, Tulane University, New Orleans, Louisiana.

*These authors contributed equally to this work.
} 


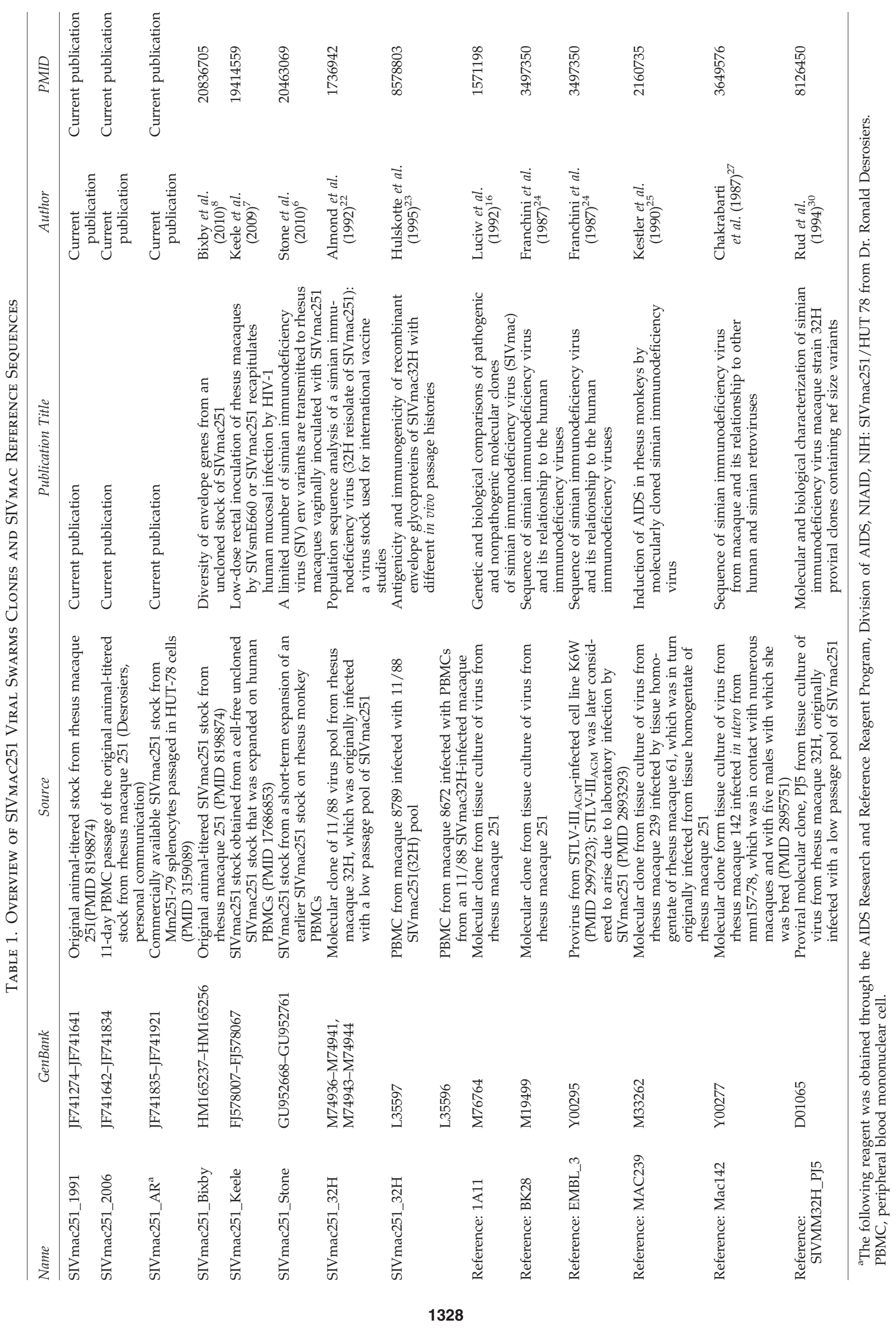


Table 1 shows an overview of the viral sequences analyzed. The SIVmac251_2006 swarm was derived by expanding an aliquot of the 1991 quasispecies in peripheral blood mononuclear cells (PBMCs) from superclean-specific pathogen-free (SPF) rhesus monkeys for 11 days in 2006 (R. Desrosiers, personal communication). The SIVmac251 available from the AIDS Reagents Program (SIVmac251_AR) was obtained by passaging splenic lymphocytes of the Mm251-79 primate in the human T cell line HUT-78. ${ }^{11}$ Bixby et al. ${ }^{8}$ cloned and sequenced the gp120 of 20 SIVmac251_1991 strains. Keele et al. ${ }^{7}$ also described 61 envelope sequences obtained from passaging splenocytes of Mm251-79 in human PBMCs, whereas the 25 sequences described by Stone et al. ${ }^{6}$ were passaged in rhesus monkey's PBMCs. Different SIVmac251_32H viral pools were obtained from new animals that were infected with an uncloned SIVmac251 quasispecies. Finally, additional reference sequences representing known SIVmac clones of different origin were also included in the analysis (Table 1).

To characterize further the genetic heterogeneity of the major SIVmac251 swarms available, total RNA from the SIVmac251_1991 and SIVmac251_2006 viral swarm was obtained from the New England Primate Research Center (courtesy of Dr. R. Desrosiers). An aliquot of SIVmac251_AR ${ }^{11}$ viral swarm was also obtained from the AIDS Research and Reference Reagent Program, Division of AIDS, NIAID, NIH, and total RNA was purified using the QIAamp Viral RNA mini kit (Qiagen). Reverse transcription was performed using the SuperScript III kit (Invitrogen, Carlsbad, CA) according to the manufacturer's recommendations. The eluted RNA was used for at least two separate reverse transcriptions using envspecific primers previously described. ${ }^{7}$ Multiple nested polymerase chain reactions (PCRs) were performed on the cDNA to amplify the full-length gp120 env gene using primers and protocol previously described. ${ }^{12}$ Amplified products were cloned using the TOPO-TA cloning kit (Invitrogen) and sequenced at the University of Florida Interdisciplinary Center for Biotechnology Research Sequencing Core facility to obtain a final data set including 368 clones of SIVmac251_1991, 193 clones of SIVmac251_2006, and 87 clones of SIVmac251_AR. Sequences were assembled with the Co-
donCode software (CodonCode Corporation, Dedham, MA) and aligned with the Clustal ${ }^{13}$ algorithm implemented in BioEdit, ${ }^{14}$ followed by a manual optimization protocol taking into account conserved glycosylation motifs. ${ }^{15}$ All alignments were gap-stripped for further analyses. The new sequences were deposited in GenBank with accession numbers JF741274-JF741921. The alignments are available from the authors upon request.

To assess for PCR and sequencing errors, PCR and cloning were performed under the same conditions using the 1A11 clone (obtained through the AIDS Research and Reference Reagent Program, Division of AIDS, NIAID, NIH: SIV $_{\text {mac }} 1$ A11 DNA from Dr. P. Luciw) initially derived from the SIVmac251 swarm as a template. ${ }^{16}$ To calculate the PCR error rate, 96 clones were sequenced, in both the forward and the reverse direction. The sequences were quality trimmed to $700 \mathrm{bp}$ and aligned using BioEdit. Using the $1 \mathrm{~A} 11$ sequence as a reference, a pairwise analysis was performed to compute the number of nucleotide differences with MEGA v4.0, ${ }^{17}$ which resulted in an overall rate of 1.2 errors/ $\mathrm{kb}(1 \%)$. All errors were due to point mutations at singleton positions and no recombinant sequences, which could result by Taq polymerase-induced template switching, were detected. To calculate the sequencing error rate, a single clone was sequenced directly 96 times, and only one error was observed overall. To correct for the PCR error rate in the viral sequences, nucleotide changes present in $\leq 1 \%$ per nucleotide position were adjusted to the nucleotide with the highest frequency.

Average within-group diversity for different SIVmac251 stocks was calculated from pairwise distance measures using uncorrected $p$-distances, and 500 bootstrap replicates to estimate the standard errors, with MEGA v4.0 (Table 2). All the swarms showed a heterogeneous viral population $(0.3-1.0 \%)$. Interestingly, the diversity of the nonpassaged $20 \mathrm{SIV}$ mac251_Bixby sequences $(1.0 \% \pm 0.4)$ was not significantly different (two-tailed $t$-test $p>0.05$ ) from that of the 368 SIVmac251_1991 sequences $(0.8 \% \pm 0.2)$, indicating that a small number of clones is already sufficient to describe the genetic heterogeneity of the original stock, although it may not capture low frequency variants that could significantly contribute

Table 2. Genetic Characterization of SIV Viral Swarm Envelope Sequences

\begin{tabular}{|c|c|c|c|c|c|c|c|}
\hline \multirow{2}{*}{$\begin{array}{l}\text { Macaque } \\
\text { SIVmac251_1991c }\end{array}$} & \multirow{2}{*}{$\begin{array}{c}\begin{array}{c}\text { Number } \\
\text { of sequences }\end{array} \\
368\end{array}$} & \multicolumn{2}{|c|}{ Mean diversity } & \multicolumn{2}{|c|}{ Mean divergence } & \multirow{2}{*}{$\frac{\text { V1 length }}{36-47}$} & \multirow{2}{*}{$\begin{array}{c}\begin{array}{c}\text { V1 N-linked } \\
\text { glycosylation sites }\end{array} \\
2-3\end{array}$} \\
\hline & & 0.8 & $(0.1)$ & - & - & & \\
\hline SIVmac251_2006 ${ }^{\mathrm{d}}$ & 193 & 0.6 & $(0.1)$ & 0.7 & $(0.1)$ & $36-47$ & $2-3$ \\
\hline SIVmac251_AR ${ }^{\mathrm{e}}$ & 87 & 0.5 & $(0.1)$ & 2.1 & $(0.4)$ & 38,41 & 2 \\
\hline SIVmac251_Keele ${ }^{\mathrm{f}}$ & 61 & 0.3 & $(0.1)$ & 1.0 & $(0.2)$ & 42 & 3 \\
\hline SIVmac251_Bixby ${ }^{\mathrm{g}}$ & 20 & 1.0 & $(0.2)$ & 0.9 & $(0.1)$ & $38-42$ & $2-3$ \\
\hline SIVmac251_Stone ${ }^{\mathrm{h}}$ & 25 & 0.3 & $(0.1)$ & 2.0 & $(0.4)$ & 42,45 & 2 \\
\hline SIVMM32H & 10 & 0.9 & $(0.1)$ & 1.8 & $(0.3)$ & $38-44$ & 2 \\
\hline
\end{tabular}

\footnotetext{
a Average nucleotide diversity ( $p$-distance) of sequences within a specific SIV viral swarm. Values are in \%; standard errors are given in parentheses.

${ }^{\mathrm{b}}$ Mean nucleotide divergence ( $p$-distance) of viral swarm sequences compared to the 1991 SIVmac251 viral swarm. Values are in \%; standard errors are given in parentheses.

c1991 viral swarm from New England Primate Center. GenBank accession numbers: JF741274-JF741641.

${ }^{\mathrm{d}}$ PBMC passaged (11 days) 1991 viral swarm from New England Primate Center. GenBank accession numbers: JF741642-JF741834.

eViral swarm from AIDS Reagent Program. GenBank accession numbers: JF741835-JF741921.

${ }^{f}$ Viral swarm from Keele et al. (2009). ${ }^{7}$ GenBank accession numbers: FJ578007-FJ578039.

${ }^{g}$ Viral swarm from Bixby et al. (2010). ${ }^{8}$ GenBank accession numbers: HM165237-HM165256.

${ }^{\mathrm{h}}$ Viral swarm from Stone et al. (2010). ${ }^{6}$ GenBank accession numbers: GU952668-GU952692.
} 


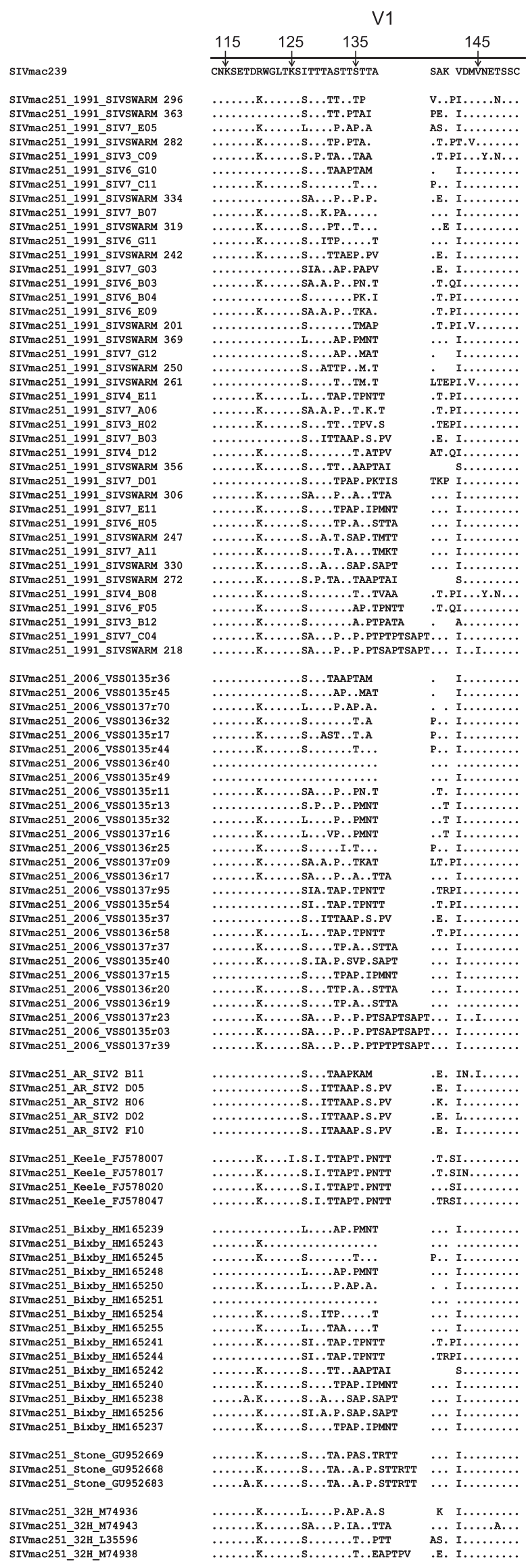

to the founder population in newly infected animals. Viral diversity was lower in the four stocks derived from passage/ expansion in vitro of SIVmac251, although it was significant only for SIVmac251_AR and SIVmac251_Keele $(p<0.05)$, while not significant $(p>0.05)$ for the SIVmac251_2006 and SIVmac251_Stone (see Table 1). Viral diversity of the animal passaged SIVmac251_32H $(0.9 \% \pm 0.1)$ was comparable to the SIVmac251_1991. For each viral swarm, the average divergence from SIVmac251_1991 was also calculated. SIVmac251_2006, SIVmac251_ Bixby, and SIVmac251_ Keele showed similar divergence (0.7-0.9\%) from SIVmac251_1991 (Table 2). On the other hand, SIVmac251_AR, SIVmac251_Stone, and SIVmac251_32H appeared to be more divergent $(1.8-2.1 \%)$. As expected, the greatest variability was found in the $\mathrm{V} 1$ region both in terms of sequence length polymorphism and amino acid (aa) replacements. In the sequences obtained from both the 1991 and the 2006 SIVmac251 swarm, the length of the V1 region ranged from 36 to 47 aa. There was a reduced length variation (38 to 42 aa) in the Bixby et al. ${ }^{8}$ sequences. Sequences of different length were also characterized by a different number of glycosylation sites and aa motifs (Fig. 1). Only two distinct length variants (38 and 41 and 42 and 45 aa, respectively) were present in SIVmac251_AR and SIVmac251_Stone, and one distinct length of 42 aa for SIVmac251_Keele.

A neighbor-joining tree was inferred, including all the sequences described in Table 1, with the maximum-likelihood composite model implemented in MEGA v4.0 (Fig. 2). Statistical support was assessed by 1000 bootstrap replicates. The similar genetic diversity of SIVmac251_1991 and SIVmac251_2006 and SIVmac251_Bixby was reflected by the star-like phylogeny, in which the sequences from each swarm appeared highly intermixed with low statistical support $(<50 \%$ bootstrap) for distinct subpopulations. The SIVmac251_AR, SIVmac251_Stone, SIVmac251_32H, and SIVmac251_Keele sequences clustered within distinct monophyletic clades, although only the SIVmac251_AR and the SIVmac251_Stone clades were significantly supported (bootstrap $>90 \%$ ).

This study is the first in-depth sequence comparison and phylogenetic characterization of different sources of SIVmac251 viral swarms. Sequence comparison and phylogenetic analysis demonstrated that the diversity and genetic composition of the original quasispecies were significantly affected by either in vitro or in vivo passage. These results highlight the importance of knowing the origin and passage history of the infecting swarm used in the SIV/macaque model. For example, if a less diverse swarm is used, immunological, pathological, and vaccine development studies may be biased. Moreover, infection with the heterogeneous SIVmac251 quasispecies constitutes a well-established model for studying neuroAIDS. ${ }^{2,18,19}$ An advantage of using a well-characterized and heterogeneous swarm is the possibility of investigating how the initial transmitting/founder viral population affects brain infection and disease progression. In general, a

FIG. 1. Alignment in the V1 region of representative sequences from the SIVmac251 viral swarm. Sequences are aligned to the SIVmac239 clone used as reference. Identical aa are indicated by a dot. Gaps are represented by blank spaces. 
FIG. 2. Neighbor-joining tree of gp120 sequences from the SIVmac251 viral swarms. Sequences were generated from stocks provided by the New England Primate Research Center (SIVmac251_ 1991, $n=368$; SIVmac251_2006, $n=193)$ and the AIDS Reagent Program (SIVmac251_ AR, $n=87)$. The SIVmac251_ Bixby, SIVmac251_Keele, SIVmac251_Stone, SIVmac251_ $32 \mathrm{H}$, and SIVmac reference sequences were downloaded from GenBank. Accession numbers are given in Table1. The tree was inferred using the maximum-likelihood composite model of nucleotide substitution. Branches are scaled in substitutions/ site according to the scale at the bottom the tree. Branches are colored according to the legend in the figure. Supported monophyletic clades (bootstraps $\geq 75 \%$ ) are indicated by a star.
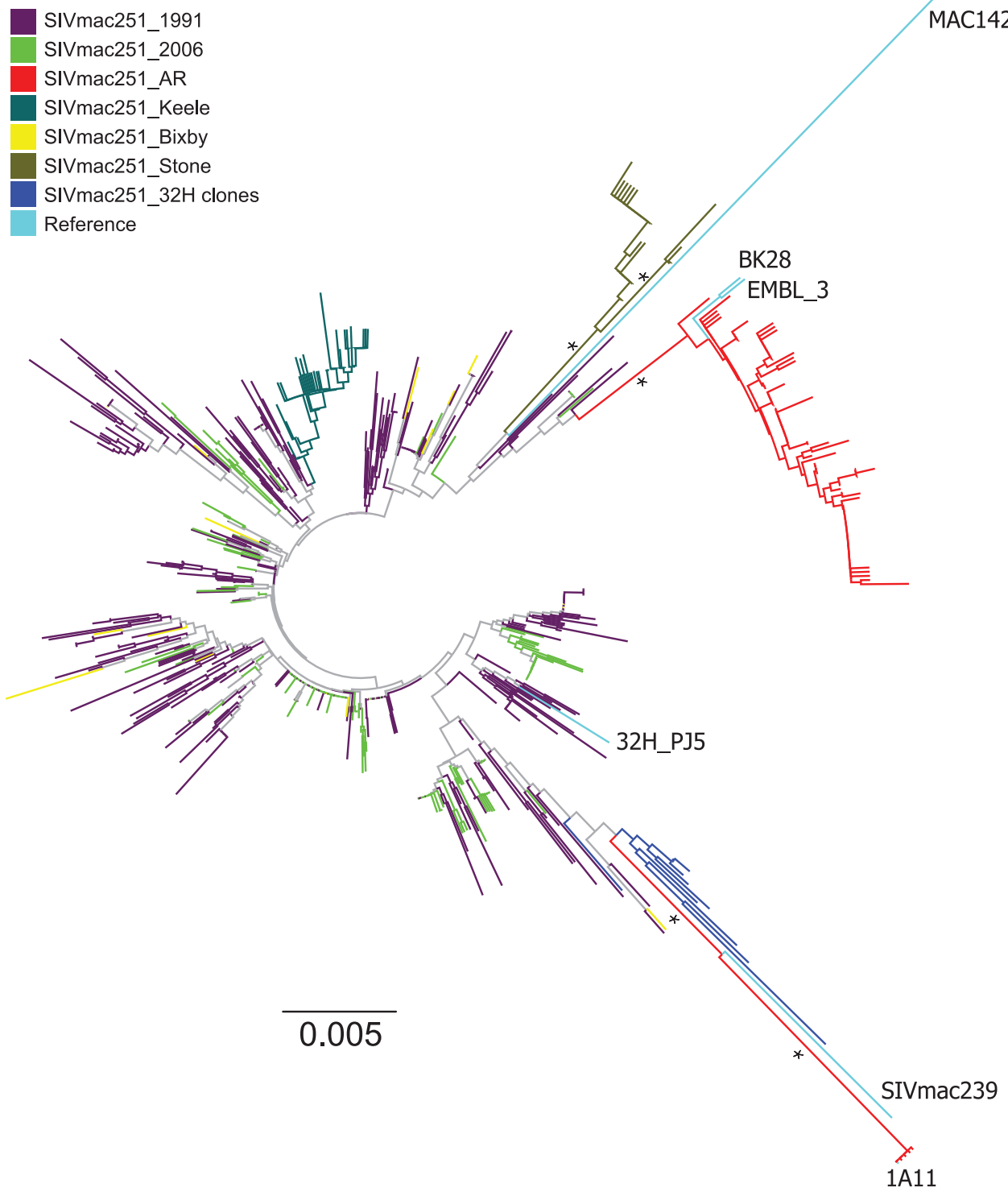

clear familiarity of the specific viral swarm used in each experiment will play a vital role in future studies by reducing confounding factors and helping to compare results among different experimental settings. ${ }^{20-30}$

The sequences were deposited in GenBank with accession numbers JF741274-JF741921.

\section{Acknowledgments}

M.S. was supported by NIH R01 NS063897-01A2 and AI065265. R.R.G. was funded through T32_NIH_CA_09126. R.R.G., S.S., and M.S. designed the study, analyzed the data, and wrote the manuscript. T.H.B, B.N., X.A., and C.C.M. performed the experiments with the animal model. D.J.N. and E.H. designed and performed the SIV sequencing project and developed the protocol to asses and correct PCR/sequencing error rate. K.W. contributed substantially to the study conception. M.M.G. and S.L.L. helped with sequence analysis. We are indebted to Amanda Lowe for assistance with the SIV sequencing and Mattia Prosperi for assistance with the sequence clean-up.
We thank Dr. Czerne Reid for assistance editing the manuscript.

\section{Author Disclosure Statement}

No competing financial interests exist.

\section{References}

1. Desrosiers RC: The simian immunodeficiency viruses. Annu Rev Immunol 1990;8:557-578.

2. Burudi EM and Fox HS: Simian immunodeficiency virus model of HIV-induced central nervous system dysfunction. Adv Virus Res 2001;56:435-468.

3. Wilson NA and Watkins DI: Is an HIV vaccine possible? Braz J Infect Dis 2009;13:304-310.

4. Simon MA, Brodie SJ, Sasseville VG, Chalifoux LV, Desrosiers RC, and Ringler DJ: Immunopathogenesis of SIVmac. Virus Res 1994;32(2):227-251.

5. Hunt RD, Blake BJ, Chalifoux LV, Sehgal PK, King NW, and Letvin NL: Transmission of naturally occurring lymphoma 
in macaque monkeys. Proc Natl Acad Sci USA 1983;80(16): 5085-5089.

6. Stone M, Keele BF, Ma ZM, et al.: A limited number of simian immunodeficiency virus (SIV) env variants are transmitted to rhesus macaques vaginally inoculated with SIVmac251. J Virol 2010;84:7083-7095.

7. Keele B, Li H, Learn G, et al.: Low-dose rectal inoculation of rhesus macaques by SIVsmE660 or SIVmac251 recapitulates human mucosal infection by HIV-1. J Exp Med 2009; 206(5):1117-1134.

8. Bixby JG, Laur O, Johnson WE, and Desrosiers RC: Diversity of envelope genes from an uncloned stock of SIVmac251. AIDS Res Hum Retroviruses 2010;26(10):1115-1131.

9. Lena P, Villinger F, Giavedoni L, Miller CJ, Rhodes G, and Luciw P: Co-immunization of rhesus macaques with plasmid vectors expressing IFN-gamma, GM-CSF, and SIV antigens enhances anti-viral humoral immunity but does not affect viremia after challenge with highly pathogenic virus. Vaccine 2002;20(Suppl 4):A69-79.

10. Feinberg MB and Moore JP: AIDS vaccine models: Challenging challenge viruses. Nat Med 2002;8:207-210.

11. Daniel MD, Letvin NL, King NW, et al.: Isolation of T-cell tropic HTLV-III-like retrovirus from macaques. Science 1985;228(4704):1201-1204.

12. Stephens EB, Liu ZQ, Zhu GW, et al.: Lymphocyte-tropic simian immunodeficiency virus causes persistent infection in the brains of rhesus monkeys. Virology 1995;213:600-614.

13. Thompson JD, Higgins DG, and Gibson TJ: CLUSTAL W: Improving the sensitivity of progressive multiple sequence alignment through sequence weighting, position-specific gap penalties and weight matrix choice. Nucleic Acids Res 1994;22(22):4673-4680.

14. Hall TA: BioEdit: A user-friendly biological sequence alignment editor and analysis program for Windows 95/98/ NT. Nucleic Acids Symp Ser 1999;22:95-98.

15. Lamers SL, Sleasman JW, and Goodenow MM: A model for alignment of Env V1 and V2 hypervariable domains from human and simian immunodeficiency viruses. AIDS Res Hum Retroviruses 1996;12(12):1169-1178.

16. Luciw PA, Shaw KE, Unger RE, et al.: Genetic and biological comparisons of pathogenic and nonpathogenic molecular clones of simian immunodeficiency virus (SIVmac). AIDS Res Hum Retroviruses 1992;8(3):395-402.

17. Tamura K, Dudley J, Nei M, and Kumar S: MEGA4: Molecular Evolutionary Genetics Analysis (MEGA) software version 4.0. Mol Biol Evol 2007;24:1596-1599.

18. Anthony IC, Ramage SN, Carnie FW, Simmonds P, and Bell JE: Influence of HAART on HIV-related CNS disease and neuroinflammation. J Neuropathol Exp Neurol 2005;64(6): 529-536.
19. Bell JE: An update on the neuropathology of HIV in the HAART era. Histopathology 2004;45:549-559.

20. Lewis MG, Bellah S, McKinnon $\mathrm{K}$, et al.: Titration and characterization of two rhesus-derived SIVmac challenge stocks. AIDS Res Hum Retroviruses 1994;10(2):213-220.

21. Letvin NL, Rao SS, Dang V, et al.: No evidence for consistent virus-specific immunity in simian immunodeficiency virusexposed, uninfected rhesus monkeys. J Virol 2007;81:1236812374.

22. Almond N, Jenkins A, Slade A, Heath A, Cranage M, and Kitchin P: Population sequence analysis of a simian immunodeficiency virus (32H reisolate of SIVmac251): A virus stock used for international vaccine studies. AIDS Res Hum Retroviruses 1992;8(1):77-88.

23. Hulskotte EG, Rimmelzwaan GF, Boes J, et al.: Antigenicity and immunogenicity of recombinant envelope glycoproteins of SIVmac32H with different in vivo passage histories. Vaccine 1995;13:1187-1197.

24. Franchini G, Gurgo C, Guo HG, et al.: Sequence of simian immunodeficiency virus and its relationship to the human immunodeficiency viruses. Nature 1987;328(6130):539-543.

25. Kestler H, Kodama T, Ringler D, et al.: Induction of AIDS in rhesus monkeys by molecularly cloned simian immunodeficiency virus. Science 1990;248(4959):1109-1112.

26. Daniel MD, Letvin NL, Sehgal PK, et al.: Prevalence of antibodies to 3 retroviruses in a captive colony of macaque monkeys. Int J Cancer 1988;41(4):601-608.

27. Chakrabarti L, Guyader M, Alizon M, et al.: Sequence of simian immunodeficiency virus from macaque and its relationship to other human and simian retroviruses. Nature 1987;328(6130):543-547.

28. Kanki PJ, Alroy J, and Essex M: Isolation of T-lymphotropic retrovirus related to HTLV-III/LAV from wild-caught African green monkeys. Science 1985;230(4728):951-954.

29. Kestler HW, 3rd, Li Y, Naidu YM, et al.: Comparison of simian immunodeficiency virus isolates. Nature 1988;331(6157): 619-622.

30. Rud EW, Cranage M, Yon J, et al.: Molecular and biological characterization of simian immunodeficiency virus macaque strain $32 \mathrm{H}$ proviral clones containing nef size variants. J Gen Virol 1994;75(Pt 3):529-543.

Address correspondence to: Marco Salemi

Department of Pathology, Immunology, and Laboratory Medicine Emerging Pathogens Institute University of Florida College of Medicine Gainesville, Florida 32610

E-mail: salemi@pathology.ufl.edu 